\title{
A Study on Acceleration Waveform Control of an Electrohydraulic Servovibrator using Direct Digital Control* (Linear-Model-Following-Control System)
}

\author{
Kunio KOBAYASHI**, Sanroku SATO** \\ and Tetsuzi SATO***
}

\begin{abstract}
In this paper, a linear-model-following control (LMFC) method using direct digital control (DDC) to reproduce the input waveform is discussed. It is difficult to control the acceleration waveform of an electrohydraulic servovibrator because of its nonlinear characteristics. To control this acceleration waveform, the LMFC method with a reference linear model of a time-delay element is introduced. This LMFC system has a state variable feedback circuit with variable feedback gains, and the calculation of the variable feedback gains is performed by means of a digital processor. The LMFC method is applied to the analogue simulation circuit and experimental apparatus to confirm reproducibility of the input signal. The experiment shows that the performance index of waveform distortion is $11 \%$ for a reference signal of triangular waveform with a fundamental frequency of $30 \mathrm{~Hz}$.
\end{abstract}

Key Words : Computer Control, Acceleration Waveform Control, Electrohydraulic Servovibrator, Reproducibility, Linear-Model-Following Control, DDC, Nonlinear Control

\section{Introduction}

It is believed that control of the output waveform of the electrohydraulic servovibrator is difficult because of its nonlinear characteristics. Therefore, some studies have been reported which address the problem $^{(1) \sim(3)}$. However, in these studies, we note that the effectiveness of control is not sufficient, the structure of the control system is complex, and so on. For waveform control of the electrohydraulic servovibrator, we have shown that the servo system controlled by means of LMFC exhibits high performance in reproduction of displacement waveforms and that the system is constructed without any difficulty, using a digital processor ${ }^{(4),(5)}$.

In this research, a design for acceleration waveform control of the electrohydraulic servovibrator is discussed. To control the acceleration

* Received 25th June, 1991

* Department of Mechanical Engineering, Musashi Institute of Technology, 1-28-1 Tamazutsumi, Setagaya-ku, Tokyo 158, Japan

*** Yamatake-Honeywell Co., Ltd., 2-12-19 Shibuya, Shibuya-ku, Tokyo 150, Japan waveform of the electrohydraulic servo system, the LMFC method with a reference linear model of a time -delay element is introduced. This LMFC system has state variable feedback circuits with variable feedback gains, and the calculation of the variable feedback gains is performed using a digital processor. The LMFC method is applied to the experimental apparatus and analogue simulation circuit to confirm reproducibility of the input signal, and the effectiveness of the control method is reported in this paper.

\section{Nomenclature}

$A$ : effective area of piston, $B$ : coefficient of viscous friction of hydraulic actuator, $D:$ derivative operator, $e(t)$ : error signal, $K$ : bulk modulus of fluid, $K_{a}$ : product of servo amplifier gain and ser. vovalve constant, $K_{d}(t n)$ : variable forward path gain, $K_{v}(t n)$ : variable feedback gain of piston velocity, $K_{a}(t n)$ : variable feedback gain of piston acceleration, $K_{y}$ : feedback gain of piston displacement, $K_{0}$ : spool valve constant, $M$ : mass of load, $p_{l}(t)$ : load pressure, $P_{s}$ : supply pressure, $q(t)$ : volumetric flow of spool valve, $r(t)$ : input signal, $s:$ Laplace operator, $u(t)$ : control signal (servovalve input), $v(t)$ : piston 
velocity, $V m$ : half volume of cylinder and conduit, $x(t)$ : spool displacement, $y(t)$ : piston displacement, $\alpha(t)$ : piston acceleration, $\delta(t n)$ : term to compensate for $d(t n), \tau$ : delay time of time-delay element, $\tilde{\varepsilon}(t)$ : acceleration error signal.

\section{Design of Linear-Model-Following-Control} System using DDC

\subsection{Dynamic equation of motion of the electro- hydraulic servomotor}

The model of the electrohydraulic servomotor is shown in Fig. 1. The dynamic equations of the electrohydraulic servomotor are represented as follows.

The relation between the input signal of the servo amplifier $e(t)$ and the spool displacement $x(t)$ is

$$
x(t)=K_{a} \cdot e(t) \text {. }
$$

The pressure-vs-flow characteristics of the spool valve can be represented by

$$
q(t)=K_{o} \cdot x(t) \sqrt{P_{s}-(\operatorname{sgn} x(t)) p_{l}(t) .}
$$

The compressibility of the fluid in the actuator is

$$
\frac{d p_{l}(t)}{d t}=\frac{2 K}{V_{m}}\left[q(t)-A \frac{d y(t)}{d t}\right],
$$

and the equation of motion of the actuator is

$$
M \frac{d^{2} y(t)}{d t^{2}}+B \frac{d y(t)}{d t}=A p_{l}(t)
$$

where $\left|p_{l}(t)\right|<P_{s}$. Equation( 2 ) can be approximately

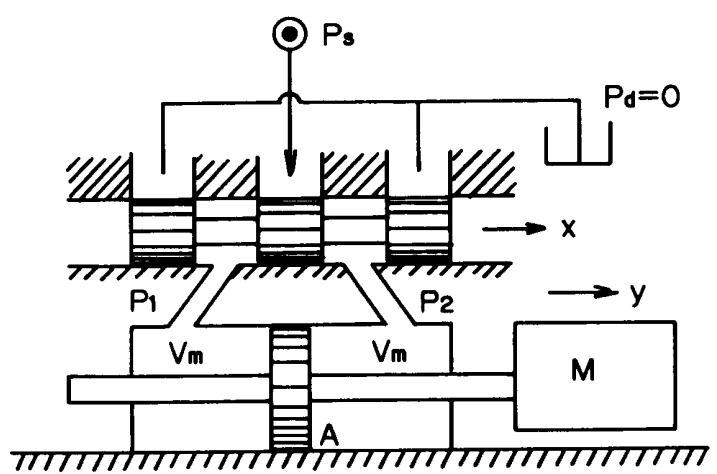

Fig. 1 Model of hydraulic servomotor

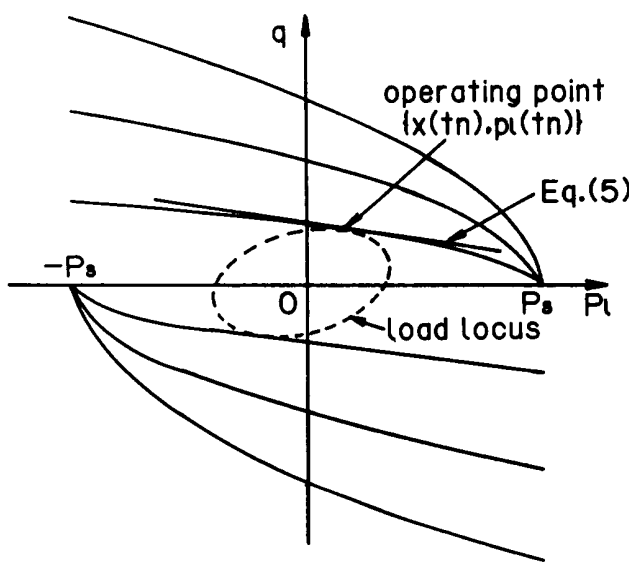

Fig. 2 Approximation of flow characteristic curve of servovalve represented by the tangent line of the pressure-vsflow curve of the spool valve at the point $[x(t n)$, $p_{l}(t n)$ ], which is sampled at $t=t n$ (see Fig. 2). In the vicinity of the operating point at $t=t n$

$$
q(t)=a \cdot x(t)-b \cdot p_{l}(t)+c
$$

where

$$
\begin{aligned}
a & =K_{o} \sqrt{P_{s}-(\operatorname{sgn} x(t n)) p_{l}(t n)}, \\
b & =\frac{K_{o}|x(t n)|}{2 \sqrt{P_{s}-(\operatorname{sgn} x(t n)) p_{l}(t n)}}, \\
c & =\frac{K_{o}|x(t n)| p_{l}(t n)}{2 \sqrt{P_{s}-(\operatorname{sgn} x(t n)) p_{l}(t n)}} .
\end{aligned}
$$

The block diagram of the electrohydraulic servomotor at the sampling instant is shown in Fig. 3. The parameters $C_{1}(t n), C_{2}(t n) K_{s}(t n)$ and $d(t n)$ are expressed in terms of $x(t n)$ and $p_{l}(t n)$, which change at every sampling instant.

$$
\begin{array}{ll}
C_{1}(t n)=\frac{B}{M}+\frac{2 K b}{V_{m}}, & C_{2}(t n)=\frac{2 K(A+B b)}{V_{m} M}, \\
K_{s}(t n)=\frac{2 K A a}{V_{m} M}, & d(t n)=\frac{c}{a} .
\end{array}
$$

The electrohydraulic servomotor shown in Fig. 3 is the object of this control system.

3.2 Structure of the acceleration waveform control system using the LMFC method

3. 2. 1 Determination of the variable gains of the state variable feedback circuits and the parameters of the series compensator In the LMFC system, the time-delay element is chosen as the ideal reference model; however, to facilitate construction of the control circuit, the 2nd-order Padé approximation ${ }^{(6)}$ of the time-delay characteristic is used. The formula of the 2nd-order Padé approximation is represented as follows :

$$
\begin{aligned}
e^{-s \tau} & \fallingdotseq \frac{\left.s^{2}-(6 / \tau) s+12 / \tau^{2}\right)}{s^{2}+(6 / \tau) s+\left(12 / \tau^{2}\right)} \\
& =\frac{s^{2}-(6 / \tau) s\left(12 / \tau^{2}\right)}{s} \cdot \frac{s}{s^{2}+(6 / \tau) s+\left(12 / \tau^{2}\right)}
\end{aligned}
$$

The former and latter parts of Eq. ( 8 ) determine the structure of the series compensator and the feedback circuits, respectively, in Fig. 4.

The motion of the electrohydraulic servomotor shown in Fig. 3 is represented as follows:

$$
\begin{gathered}
\frac{d^{2} \alpha(t)}{d t^{2}}+C_{1}(t n) \frac{d \alpha(t)}{d t}+C_{2}(t n) \alpha(t) \\
=\frac{d\left\{K_{s}(t n)\left[K_{a} u(t)+d(t n)\right]\right\}}{d t} .
\end{gathered}
$$

The input $u(t)$ of the controlled object is given by the state variable feedback as follows:

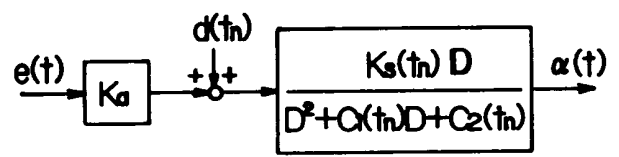

Fig. 3 Block diagram of the electrohydraulic servomotor 


$$
\begin{aligned}
& u(t)=\left\{e(t)-\left[K_{a}(t n) \frac{d v(t)}{d t}\right.\right. \\
& \left.\left.+K_{v}(t n) v(t)\right]\right\} K_{d}(t n)+\delta(t n) .
\end{aligned}
$$

Arranging the reference model given in Eq. ( 8 ), the equation of the reference model of the controlled object, which is expressed by Eq. ( 9 ), and Eq. (10) is represented as follows :

$$
\frac{d^{2} \hat{\alpha}(t)}{d t^{2}}+\left(\frac{6}{\tau}\right) \frac{d \hat{\alpha}(t)}{d t}+\left(\frac{12}{\tau^{2}}\right) \hat{\alpha}(t)=\frac{d e(t)}{d t} .
$$

Equation(11) corresponds to the latter part of Eq. (8). Let $\widehat{\varepsilon}(t)=\widehat{\alpha}(t)-\alpha(t)$; eliminating $\hat{\alpha}(t)$ and $u(t)$, Eqs. (10),(11), and (9) lead to

$$
\begin{aligned}
& \frac{d^{2} \hat{\varepsilon}(t)}{d t^{2}}+\left(\frac{6}{\tau}\right) \frac{d \hat{\varepsilon}(t)}{d t}+\left(\frac{12}{\tau^{2}}\right) \tilde{\varepsilon}(t) \\
& \quad=\frac{d}{d t}\left\{\left[C_{1}(t n)-\frac{6}{\tau}+K_{a} K_{s}(t n) K_{d}(t n) K_{a}(t n)\right] \frac{d v(t)}{d t}\right. \\
& \quad+\left[C_{2}(t n)-\frac{12}{\tau^{2}}+K_{a} K_{s}(t n) K_{d}(t n) K_{v}(t n)\right] v(t) \\
& \quad+\left[1-K_{a} K_{s}(t n) K_{d}(t n)\right] e(t) \\
& \left.\quad-K_{s}(t n)\left[d(t n)+K_{a} \delta(t n)\right]\right\},
\end{aligned}
$$

where $n=1,2,3, \cdots$ and $t_{1}, t_{2}, t_{3}, \cdots$ are sampling instants.

Because the system of Eq. (11) is stable, $\widehat{\varepsilon}(t)$ approches to zero if the right-hand side of Eq. (12) remains zero. From this condition, the control law of the variable gains $K_{\alpha}(t n), K_{v}(t n), K_{d}(t n)$, and the signal $\delta(t n)$ is given as follows:

$$
\begin{array}{ll}
K_{a}(t n)=\frac{6}{\tau}-C_{1}(t n), & K_{v}(t n)=\frac{12}{\tau^{2}}-C_{2}(t n), \\
K_{d}(t n)=\frac{1}{K_{a} K_{s}(t n)}, & \delta(t n)=-\frac{d(t n)}{K_{a}} .
\end{array}
$$

$K_{\alpha}(t n)$ and $K_{v}(t n)$ are the variable feedback gains of acceleration and velocity, respectively, $K_{d}(t n)$ is the variable gain of the forward path, and $\delta(t n)$ is the term to compensate $d(t n)$ which is shown in Fig. 3. The former part of Eq. ( 8 ) determines the structure of the series compensator.

On the other hand, in this control system the difference signal between the output signal of the time -delay model and the output acceleration signal of the hydraulic servomotor, which is referred to as the acceleration error signal, is also fed back (Fig. 4).

3.2.2 A shortening of sampling interval If it is assumed that a sampling interval is very short, we can consider $p_{l}(t) \fallingdotseq p_{l}(t n)$. Then $a, b$, and $c$ of Eq. (6) are represented as follows :

$$
a=K_{o} \sqrt{P_{s}-(\operatorname{sgn} x(t n)) p_{l}(t n),} b=0, c=0
$$

The relationship between the values of " $a$ " and " $x(t n), p_{l}(t n) "$ is input in advance as a table in the memory of the personal computer before control is initiated and the value of " $a$ " corresponding to the values of $x(t n)$ and $p_{l}(t n)$ is used at every sampling instant in the calculation of the control law by selecting it from memory. By this calculation method, the calculation time interval is improved from $2 \mathrm{msec}$ to $0.2 \mathrm{msec}$.

\section{3 Compensators}

\section{(a) Series compensator}

The signal transfer characteristic of the compensator is determined by the former part of Eq. ( 8 ) and expressed by Eq. (15). An element of derivation of Eq. (15) is composed of electric circuits approximately.

$$
G_{1}(s)=\frac{s^{2}-(6 / \tau) s+\left(12 / \tau^{2}\right)}{s}
$$

where $\tau=0.005[\mathrm{sec}]$.

(b) Dynamic compensator

This is a compensator to equate the dynamic characteristics of the servovalve to those of a proportional element and relevant to the parameter $K_{a}$ of the controlled object, which is shown in Fig. 3.

$$
G_{k}(s)=\frac{s^{2}+2 \zeta \omega_{n} s+\omega_{n}^{2}}{s^{2}+2 \zeta \alpha \omega_{n} s+\left(\alpha \omega_{n}\right)^{2}},
$$

where $\omega_{n}=565[\mathrm{rad} / \mathrm{s}], \zeta=1.2, \alpha=5$.

(c) Error compensator

The acceleration error compensator is composed of a lead-lag circuit, and parameters of the circuit are

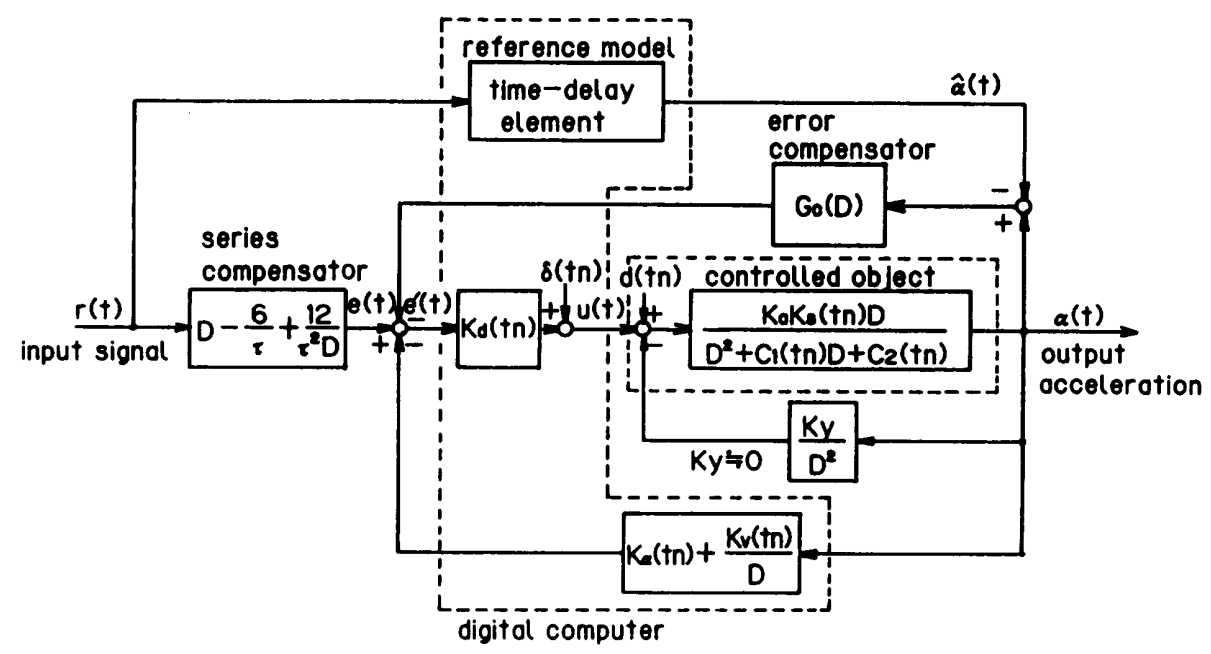

Fig. 4 Block diagram of the LMFC of the electrohydraulic servo system 
determined using the measured frequency characteristic between the signal $e(t)$ and the signal $\alpha(t)$ in Fig. 4. This is represented as follows:

$$
G_{c}(s)=\frac{1+T_{l d} \cdot s}{1+A_{l d} \cdot T_{l d} \cdot s} \cdot \frac{1+T_{l g} \cdot s}{1+A_{l g} \cdot T_{l g} \cdot s}
$$

where $T_{l d}=0.007[\mathrm{sec}], A_{l d}=0.03, T_{l \theta}=0.007$ and $A_{l g}$ $=2.5$.

\subsection{Determination of delay time of reference time-delay model}

The delay time is selected to satisfy the following two conditions :

(1) the control system has high-grade reproducibility relative to the acceleration waveform, and

(2) a higher allowable peak value of the acceleration waveform can be realized in the required frequency domain $(10 \sim 100 \mathrm{~Hz})$.

Curves of the performance index, which shows the grade of reproducibility, and the allowable peak value of the acceleration waveform are calculated with delay time as a parameter and shown in Fig. 5.

The performance index is defined to evaluate the waveform distortion and reproducibility as follows:

$$
J=\sqrt{\frac{\int_{0}^{L}[r(t)-\alpha(t-p)]^{2} d t}{\int_{0}^{L} r(t)^{2} d t}} \times 100 \%,
$$

where $\rho$ is chosen to minimize the value of $J$, and $L$ is the duration of observation. In the case of the periodic reference signal, the performance index is represented as follows :

$$
\frac{\sqrt{\sum_{n=1}^{\infty} R n^{2}\left[1+M(j n \omega)^{2}-2 M(j n \omega) \cos \left(-\psi(j n \omega)-n \omega \rho_{0}\right)\right]}}{\sqrt{\sum_{n=1}^{\infty} R n^{2}}}
$$

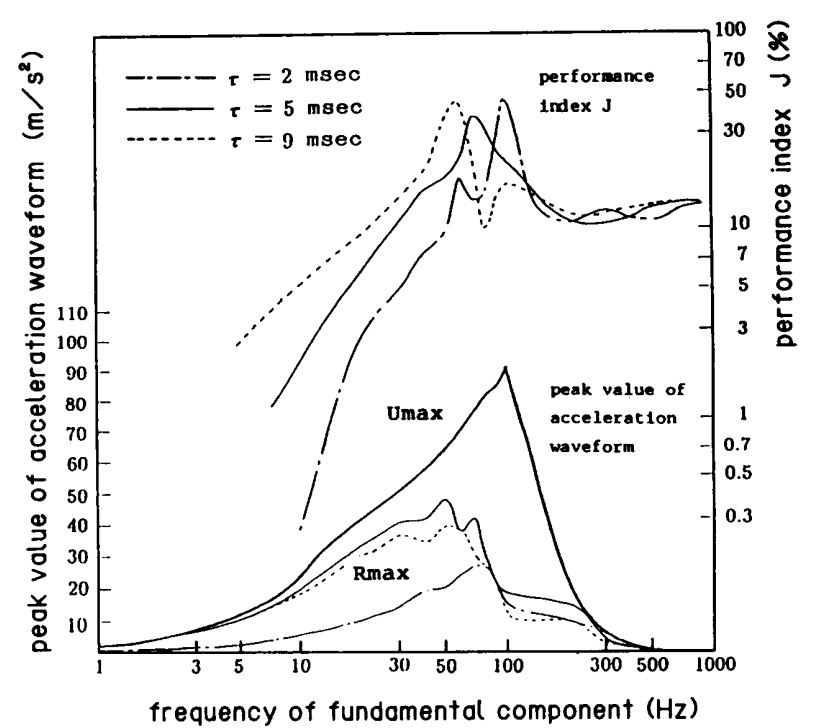

Fig. 5 The capacity and performance index of LMFC system (triangular input)

$$
\times 100(\%),
$$

where $\omega=2 \pi / L ; L$ is the period of the periodic reference signal; $M(j n \omega)$ is the gain of the LMFC system; $\phi(j n \omega)$ is the phase shift of the LMFC system; $R n$ is the amplitude of the $n$th harmonic of the input signal; $\rho_{0}$ is the adjustable phase shift to determine the minimum value of $J$.

The calculation method for the allowable peak value of the acceleration waveform is as follows. The curve of $R \max$ shows the allowable peak value of the reference signal under the limitation of the signals in the control circuit which contains an $A / D, D / A$ converter and the compensators, and it is calculated using Eqs. (1),( 3 ), (4),(13), and the compensator equations. In the calculation, the linearized model of Eq. (2) is also applied.

The curve of $U \max$ shows the allowable peak value of the output acceleration under the limitation of the rated current of the servo amplifier in the electrohydraulic servomotor. In the ideal LMFC system, it is desirable that the performance index be as small as possible and that the area enclosed by the allowable peak value be as large as possible over the required frequency domain. Therefore, the delay time of $5 \mathrm{msec}$ is selected from the figures in Fig. 5 to satisfy the above two conditions.

\section{Experiment}

\section{1 Experimental apparatus and analogue simulation circuit}

The schematic diagram of the experimental apparatus is shown in Fig. 6. In this figure, $K_{a}(t n), K_{v}(t n)$ and $K_{d}(t n)$ are calculated by the control law (Eq. (13)). The manipulating signal $u(t)$, which is generated in the personal computer, is transmitted through the D/A converter. The values of $K_{a}(t n)$ and $K_{v}(t n)$ are constant; therefore, the analogue feedback signal $V_{f}(t)$, which is the sum of the output acceleration and

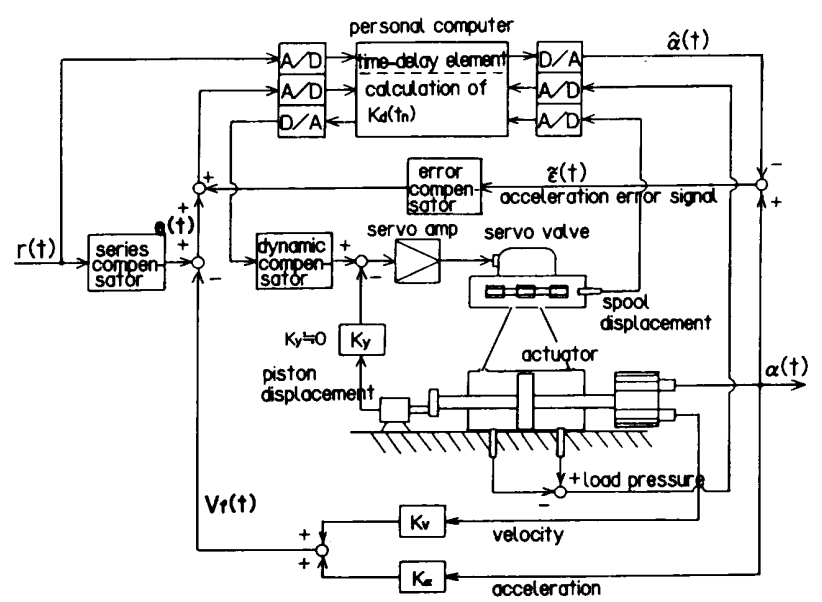

Fig. 6 Schematic diagram of experimental apparatus 
velocity signals, is fed back to the signal $e(t)$. The memory movement of a personal computer provides the physical model to produce the output signal of the ideal time-delay element.

A diagram of the analogue simulation circuit is shown in Fig. 7. In the analogue simulation circuit, the equations of motion, Eqs. $(1) \sim(4)$, are used.

(a) Construction of the personal computer system

The personal computer system consists of the cpu ( $\mu$ PD 70116-10), main memory $(640 \mathrm{~kb}), \mathrm{A} / \mathrm{D}$ con- verter ( $8 \mathrm{ch}, 12 \mathrm{bit}$ ),D/A converter ( $4 \mathrm{ch}, 12 \mathrm{bit}$ ), and a keyboard. The quantized unit of the $\mathrm{A} / \mathrm{D}$ and $\mathrm{D} / \mathrm{A}$ converters is $10 / 2048$ (volt/bit).

(b) Dimensions of the electrohydraulic servo system

The dimensions of the electrohydraulic servo system are $A=6.43\left[\mathrm{~cm}^{2}\right], B=1.145 \times 10^{3}\left[\mathrm{~N} \cdot \mathrm{s} \cdot \mathrm{m}^{-1}\right], K$ $=1.37\left[\mathrm{GN} \cdot \mathrm{m}^{-2}\right], \quad K_{0}=5.11 \times 10^{-4}\left[\mathrm{~N}^{-1 / 2} \cdot \mathrm{s}^{2} \cdot \mathrm{m}^{3}\right], \quad K_{a}=$ $0.5\left[\mathrm{~cm} \cdot \mathrm{volt}^{-1}\right], M=54[\mathrm{~kg}], P_{s}=6.86[\mathrm{MPa}]$, and $V_{m}=$ $43\left[\mathrm{~cm}^{3}\right]$. Specifications of the servovalve are a flow rate of $17.3[1 / \mathrm{min}]$ and bandwidth of $70 \mathrm{~Hz}$ for a
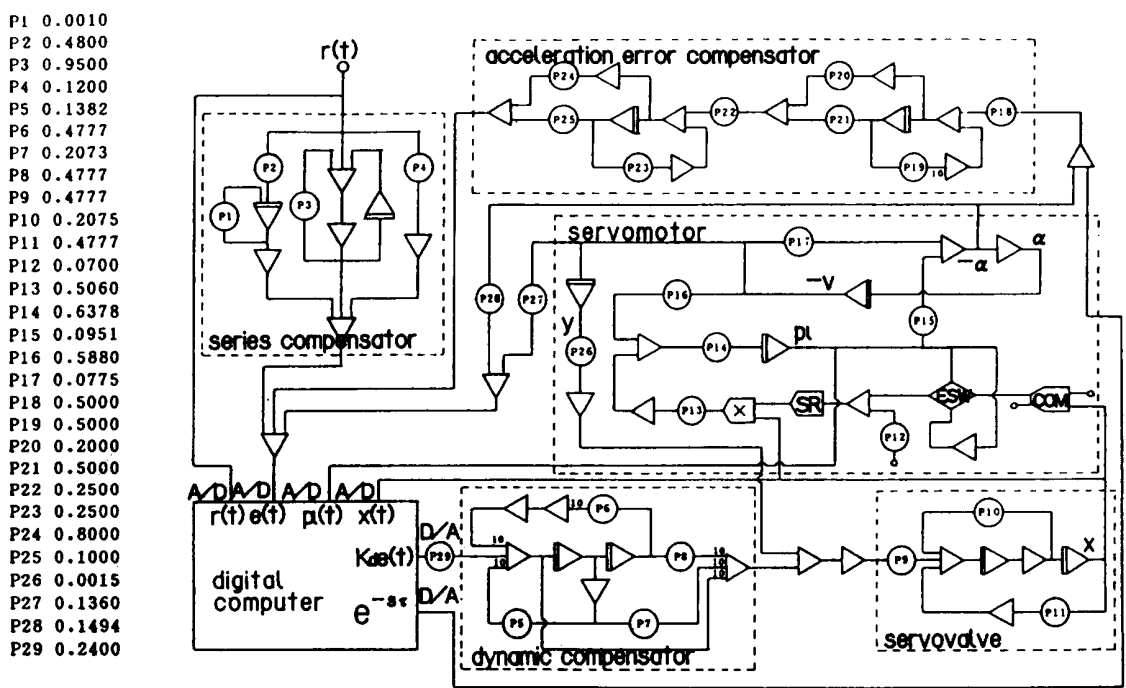

Fig. 7 Analogue simulation circuit

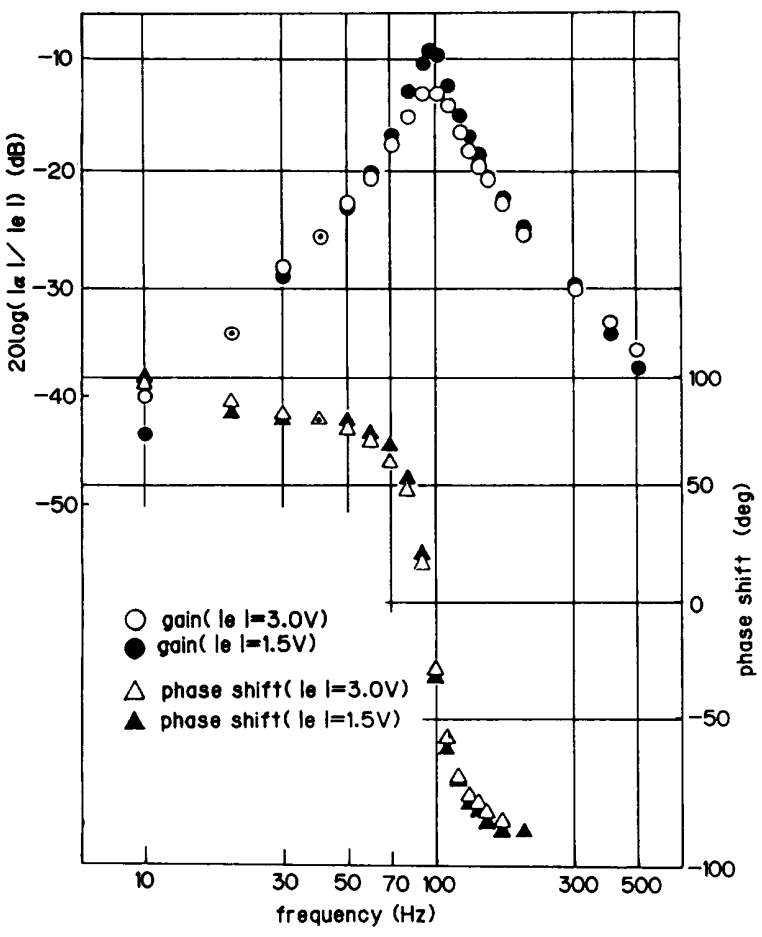

(a) without state variable feedback

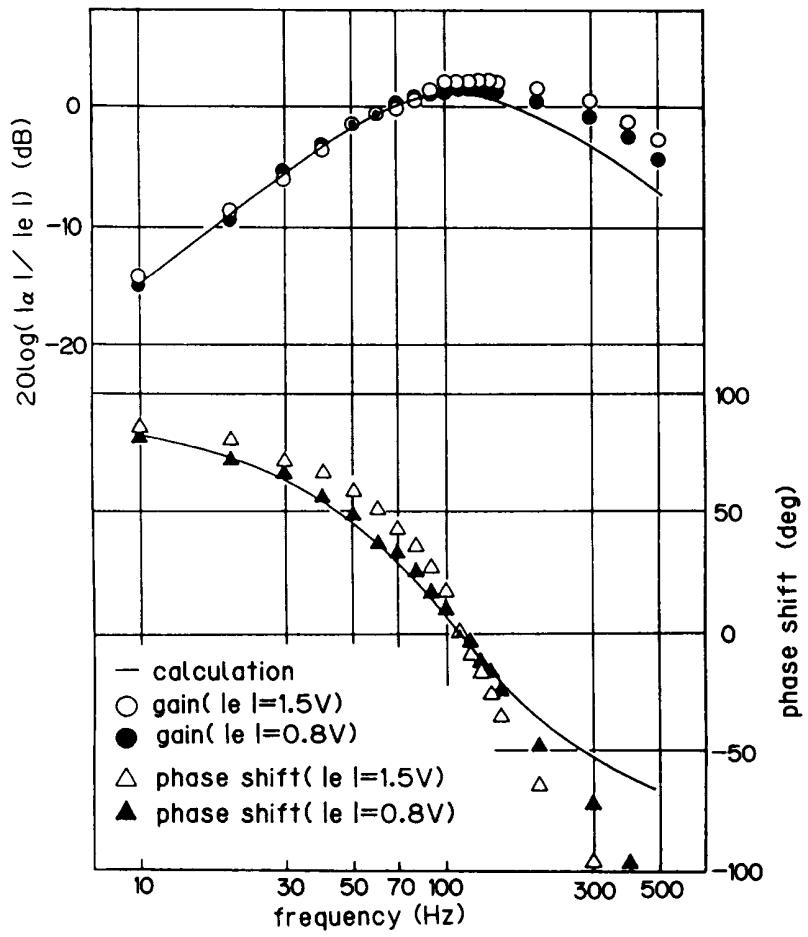

(b) with state variable feedback

Fig. 8 Frequency responses of the electrohydraulic servomotor for the analogue simulation circuit 
supplied pressure of 6.86 [MPa].

\subsection{Experimental results}

4.2.1 Control results for the analogue simulation circuit The frequency responses of the controlled object with and without state variable feedback are shown in Fig. 8. From the date shown in Fig. 8 (a ), the input amplitude dependency due to the nonlinear characteristics of the spool valve is observed, and is improved by the control as shown in Fig. 8 (b). The frequency responses of the LMFC system

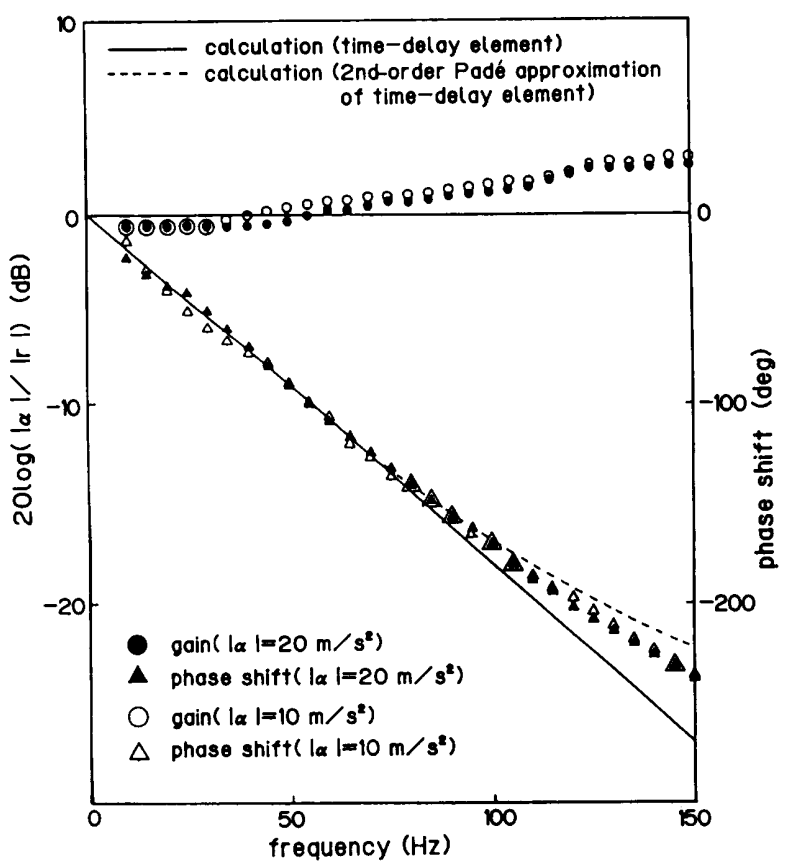

(a) without acceleration error signal feedback with and without acceleration error signal feedback are shown in Fig. 9. From these figures, the effectiveness of the acceleration error signal feedback is clearly observed. Response output acceleration waveforms for the sinusoidal and triangular inputs are shown in Fig. 10.

The experimental results of the LMFC system of the analogue simulation show satisfactory performance for the sinusoidal input signal with a frequency of $30 \mathrm{~Hz}, J=4 \%$ and for the triangular input signal

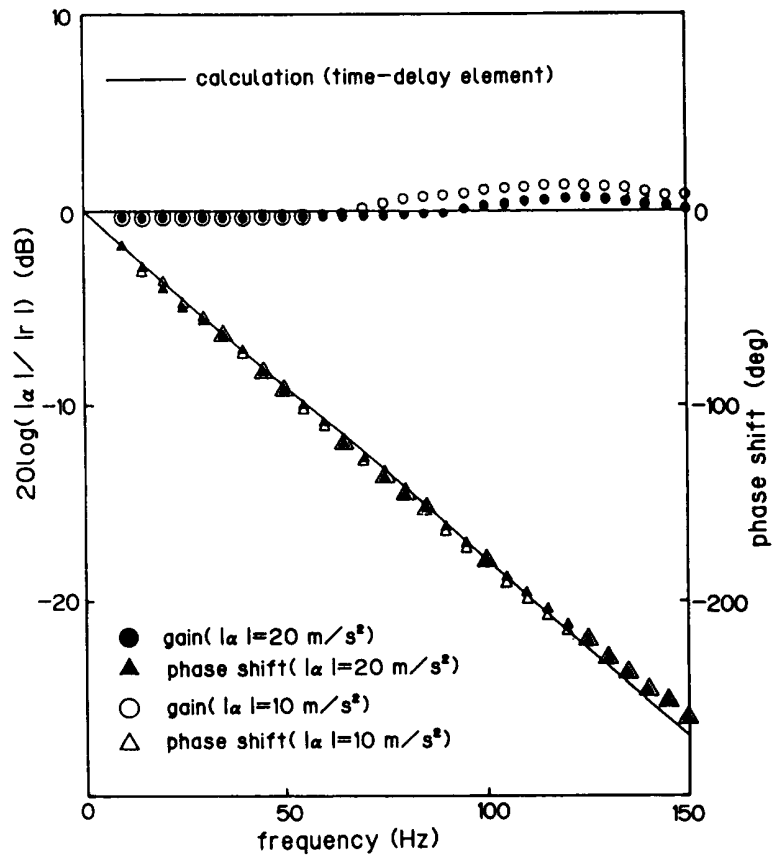

(b) with acceleration error signal feedback

Fig. 9 Frequency responses of the LMFC system for the analogue simulation circuit

$30 \mathrm{~Hz}$
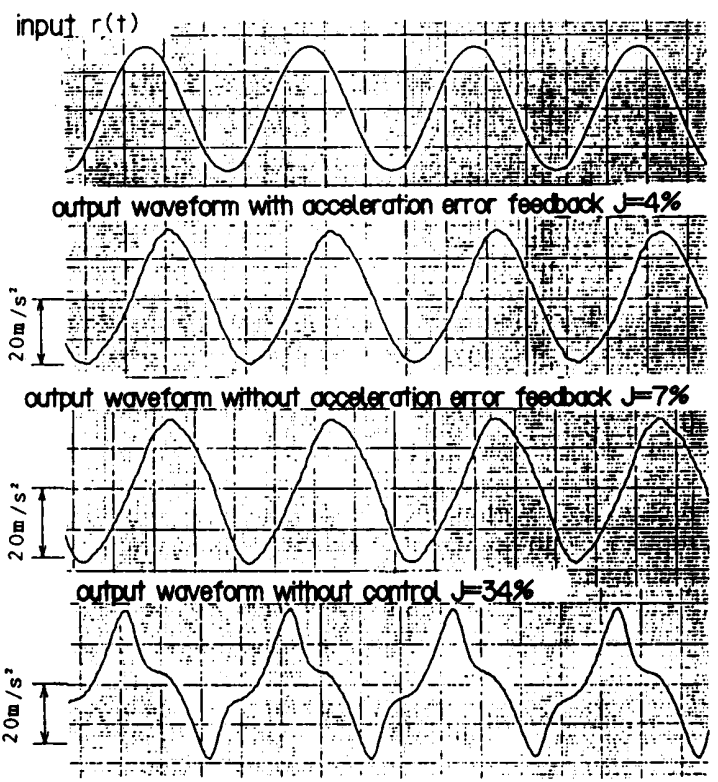

$30 \mathrm{~Hz}$

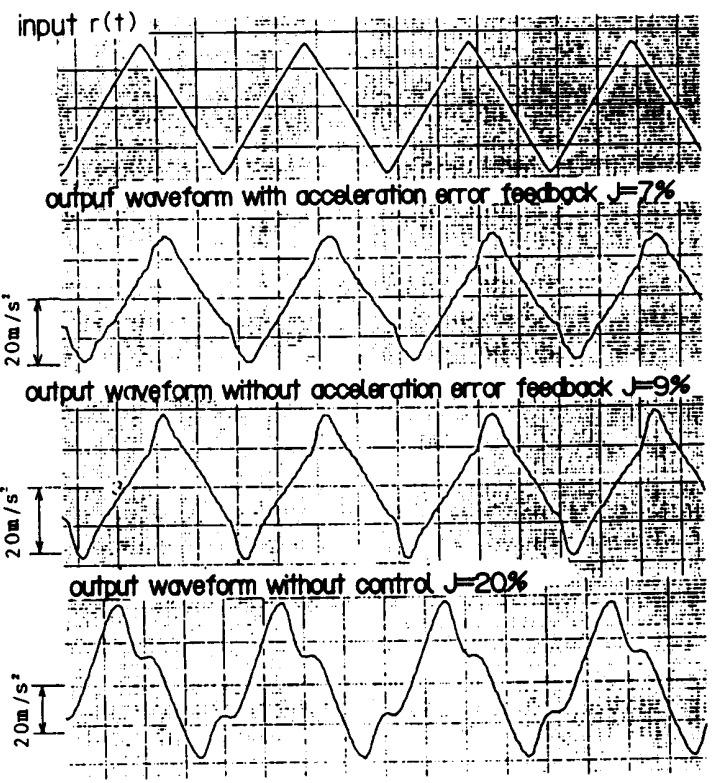

Fig. 10 Response waveforms of the LMFC system for the analogue simulation circuit 
with a fundamental frequency of $30 \mathrm{~Hz}, J=7 \%$.

4.2.2 Control results for the experimental apparatus The frequency responses of the LMFC system with and without acceleration error signal feedback are shown in Fig. 11. The experimental results of the frequency response with acceleration error signal feedback are in good agreement with the calculated results of the time-delay model, and clearly show the effectiveness of the feedback.

The experimental results for the LMFC system indicate good performance in Fig. $12 ; J=7 \%$ for the sinusoidal input signal with a frequency of $30 \mathrm{~Hz}$, and
$J=11 \%$ for the triangular input signal with a fundamental frequency of $30 \mathrm{~Hz}$. Finally, the response acceleration waveform for the eathquack waveform input is shown with good agreement in Fig. 13.

\section{Conclusions}

For application to the electrohydraulic servovibrator, the design method and the effects of the LMFC system have been presented and are listed as follows :

(1) The ideal model of LMFC, which is applied to the system to reproduce the input waveform, uses a time-delay element. To facilitate construction of the

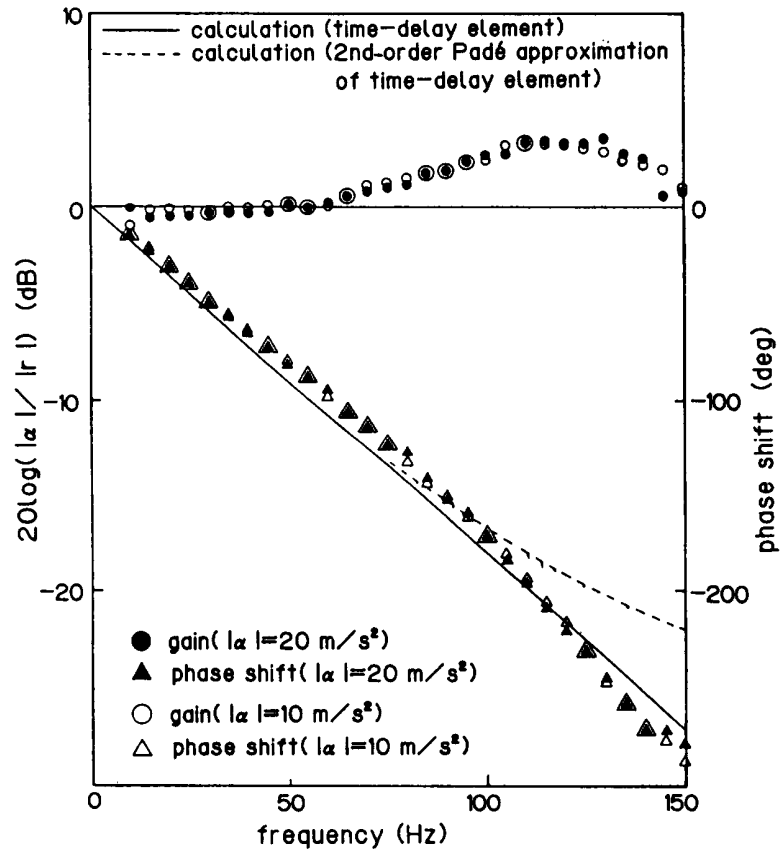

(a) without acceleration error signal feedback

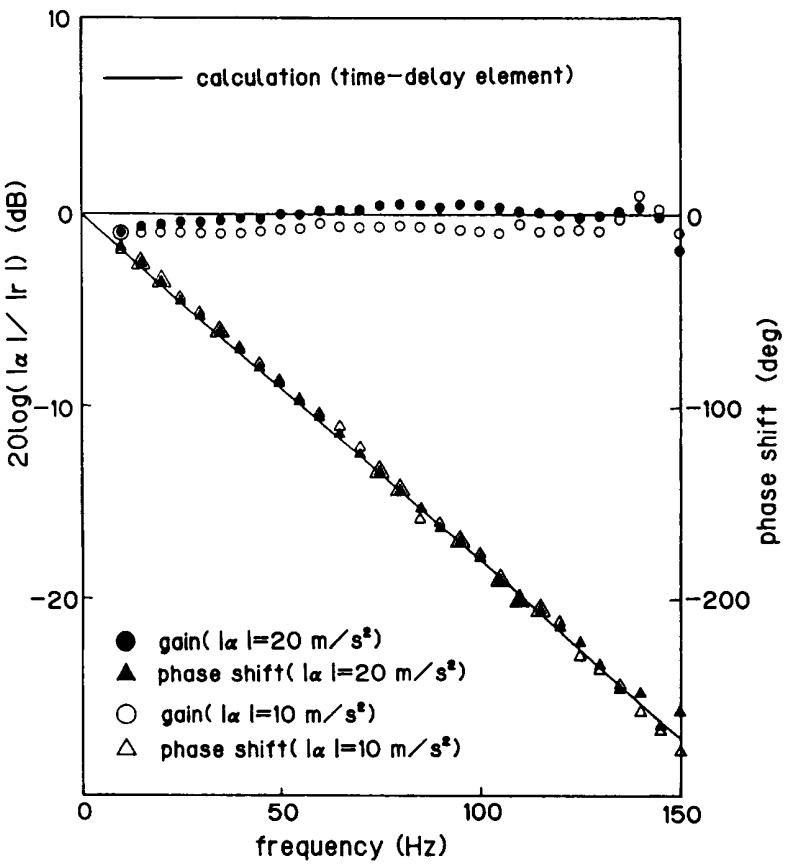

(b) with acceleration error signal feedback

Fig. 11 Frequency responses of the LMFC system for the experimental apparatus

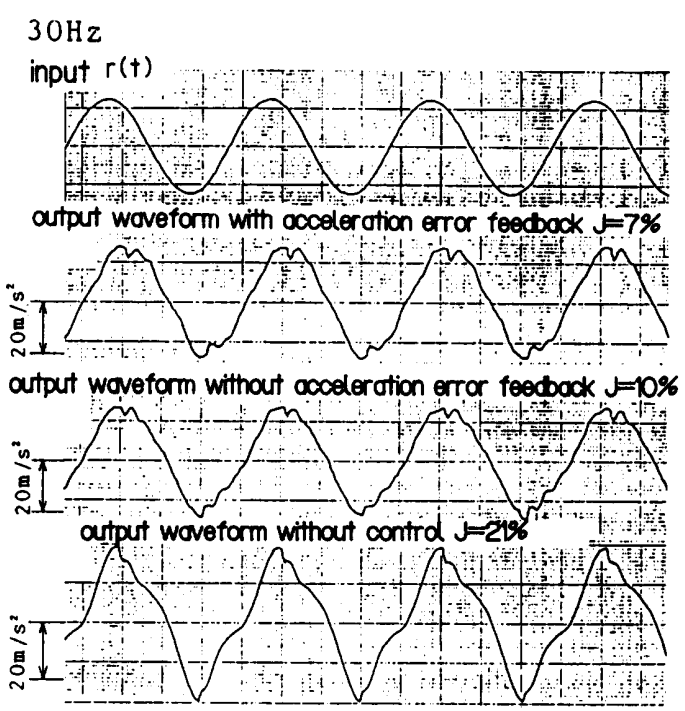

$30 \mathrm{~Hz}$

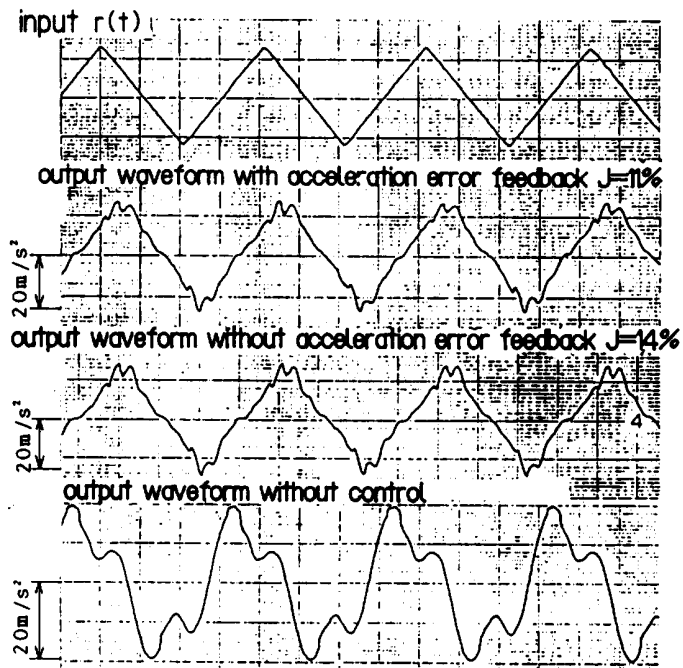

Fig. 12 Response waveforms of the LMFC system for the experimental apparatus 


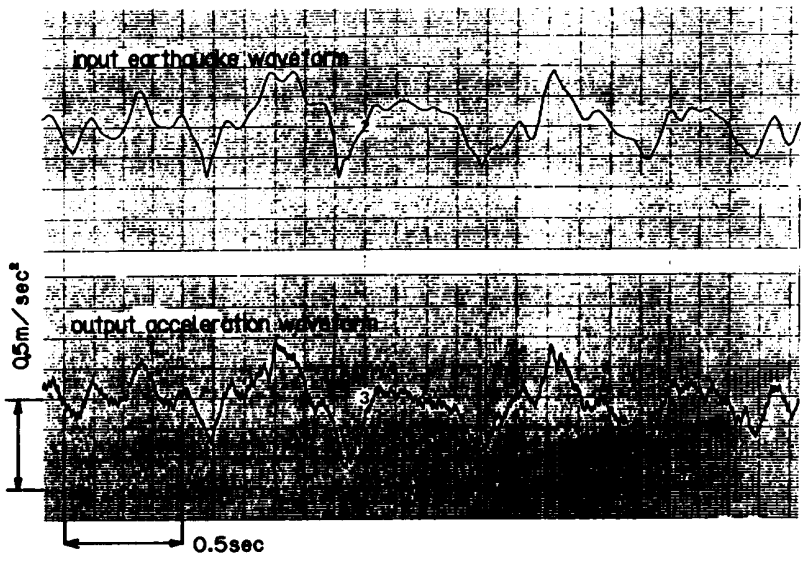

Fig. 13 Response of the earthquake waveform input for the experimental apparatus

control circuit, the 2nd-order Padé approximation of the time-delay element is available for LMFC of the electrohydraulic servo system.

(2) Theoretically, it is possible to realize LMFC for a nonlinear controlled object by means of the state variable feedback with variable gain. It is possible to realize LMFC of the electrohydraulic servo system through the use of a digital processor.

(3) With shortened sampling intervals, the calculation time of the variable gains of the state variable feedback in the digital processor becomes short enough to satisfy the stability condition of the system.

(4) The LMFC system with the acceleration error signal feedback is easily constructed. The acceleration error signal feedback compensates for the error between the ideal model and the approximate model, the error of modelling of the controlled object, the distortion due to Coulomb friction, etc.

(5) The performance index $J$ is defined to evaluate the waveform distortion and the reproducibility of the system. The allowable peak value of the output waveform of the control system is calculated and shown in Fig. 5. A suitable delay time is determined from the index $J$ and the allowable peak value.

(6) Experimental results of the LMFC system of the practical apparatus show satisfactory performance for the triangular input signal with a fundamental frequency of $30 \mathrm{~Hz}, J=11 \%$.

\section{References}

(1) Ikebe, Y. and Fukuma, K., Dynamic Load Testing Machine for Architectural Materials applying Automatic Control Techniques, Jour. SIEC, (in Japanese), Vol. 6, No. 9 (1967), p. 622.

(2) Sato, S., Fundamental Research on an Electrohydraulic-Type Acceleration Generator, Trans. SIEC, (in Japanese), Vol. 9, No. 2 (1973), p. 144.

(3) Matsuzaki, J. and Hirai, H., Research on an Electrohydraulic Shaking Machine, Trans. Jpn. Soc. Mech. Eng, (in Japanese), Vol. 42, No. 353 (1975), p. 146.

(4) Sato, S. and Kobayashi, K., A Study on DDC of Electrohydraulic Servo System, Jour. JHPS, (in Japanese), Vol. 16, No. 1 (1985), p. 55.

(5) Sato, S. and Kobayashi, K., Linear Model Follow -up Control of an Electrohydraulic Servo System by D.D.C, Jour. JHPS, (in Japanese), Vol. 18, No. 1 (1987), p. 69.

(6) Pade, H., Sur la Representation Approchee d'Unefonction par des Fractions Rationnelles, Ann. Sci. Ecole. Norm. Sup, Vol. 3, No. 9 (1892), p. 1. 